\title{
Discours
}

Revue de linguistique, psycholinguistique et

informatique. A journal of linguistics, psycholinguistics and computational linguistics

$8 \mid 2011$

Approches fonctionnelles de la structuration des textes

\section{Les emplois du marqueur discursif « di va » en ancien français}

\section{Evelyne Oppermann-Marsaux}

\section{OpenEdition}

\section{Journals}

Édition électronique

URL : http://journals.openedition.org/discours/8321

DOI : 10.4000/discours.8321

ISSN : 1963-1723

Éditeur :

Laboratoire LATTICE, Presses universitaires de Caen

Référence électronique

Evelyne Oppermann-Marsaux, "Les emplois du marqueur discursif « di va » en ancien français », Discours [En ligne], 8 | 2011, mis en ligne le 11 juillet 2011, consulté le 19 avril 2019. URL : http:// journals.openedition.org/discours/8321; DOI : 10.4000/discours.8321 

Revue de linguistique, psycholinguistique et informatique

\section{Les emplois du marqueur discursif « di va » en ancien français}

Evelyne Oppermann-Marsaux

CLESTHIA - Syled

Université de la Sorbonne Nouvelle (Paris III) 



\section{Les emplois du marqueur discursif « di va » en ancien français}

Evelyne Oppermann-Marsaux

CLESTHIA - Syled

Université de la Sorbonne Nouvelle (Paris III)

Cet article porte sur les différents emplois du marqueur discursif « di va» en ancien français. II s'intéresse plus particulièrement à trois aspects: le figement de la séquence «di va» en comparaison avec les occurrences de «va + di», la pragmaticalisation de l'impératif « di » ainsi que la position et la fonction de « di va» à l'intérieur du discours rapporté. L'étude permet de définir « di va » comme un marqueur discursif polysémique, en distinguant entre les emplois de «di va 1 », dans lesquels le sémantisme premier du verbe « dire» reste présent, et ceux de «di va 2 », où ce sémantisme s'efface. Dans ce dernier cas, « di va» devient compatible avec des contextes injonctifs et déclaratifs. Toujours employé en position initiale ( $\mathrm{Di}$ va, P»), il traduit souvent une réaction de la part du locuteur face au comportement - verbal ou non verbal - de son allocutaire et peut alors prendre des valeurs expressives différentes, allant de I'opposition à la bienveillance et à l'incrédulité. «Di va 1 » en revanche, figure exclusivement dans le contexte immédiat d'une interrogation (qu'il ne précède pas obligatoirement) et se comporte alors «seulement» comme une interjection d'appel, qui attire l'attention de l'allocutaire sur la question posée.

Mots clés: ancien français, discours direct, impératif, interjection, marqueur discursif, pragmaticalisation

This paper deals with the different uses of the discourse marker "diva" in Old French. It focuses on three main points: the fixation of the sequence "di va" compared to the occurrences of "va + di", the pragmaticalization of the imperative "di", and the position and the function of "diva" within reported speech. The study defines "di va" as a polysemic discourse marker, and distinguishes between the uses of "diva 1", where the primary lexical meaning of the verb "dire" is still present, and those of "di $v a 2^{\prime \prime}$, where this lexical meaning has disappeared. In this case, "diva" may occur in injunctive and assertive contexts. "Diva 2, $P$ " is always used in initial position, and frequently represents the speaker's reaction to the previous discourse or attitude of the addressee. It can then adopt several expressive values, ranging from denial to benevolence and disbelief. "Diva 1" on the other hand always appears in the immediate context of a question (which it does not necessarily precede), and thus behaves as an interjection which is only used to call the addressee's attention to the speaker's question.

Keywords: Old French, direct speech, imperative, interjection, discourse marker, pragmaticalization

L'étude de marqueurs discursifs dans des états anciens du français peut correspondre à une recherche des origines de marqueurs devenus usuels dans la langue moderne et à la description de la grammaticalisation progressive de ces formes au fil du temps. Telle est notamment notre perspective d'analyse dans OppermannMarsaux (2008 et 2010), articles consacrés à la naissance et au développement de marqueurs discursifs d'origine verbale, respectivement «tiens/tenez» (2008) et «allons» (2010). 
L'objet de cette étude ${ }^{1}$ est différent. Nous allons en effet nous intéresser à un marqueur discursif caractéristique de l'ancienne langue, plus particulièrement de l'ancien français des XII ${ }^{e}-\mathrm{XIII}{ }^{\mathrm{e}}$ siècles, et qui ne s'est pas conservé en français moderne : il s'agit de «di va», qui correspond, selon les descriptions qu'en donnent les dictionnaires et les manuels d'ancien français, à ce que l'on pourrait appeler une «interjection d'appel ${ }^{2}$, c'est-à-dire à une interjection qui sert à interpeller l'interlocuteur et à attirer son attention sur quelque chose:

Diva interj. ([...] juxtaposition de deux impératifs di, de dire, et va, de aller). Interjection d'encouragement, d'étonnement, allons! dis donc! (Greimas, 1980: 194)

Di va > diva. Impératif attirant l'attention, comme le fait «dis donc» en français contemporain [...]. (Buridant, 2000: 737, § 629)

\section{Les premières interrogations}

Ces deux citations soulèvent d'entrée au moins deux questions concernant l'analyse de cette forme «di va».

Tout d'abord, on peut se demander si «di va» correspond toujours à un marqueur unique, comme le suggère le titre de cet article, ou s'il peut, du moins dans certains cas, être décrit comme une juxtaposition de deux unités distinctes. Si nos définitions soulignent bien que «di va» est employé comme une interjection, elles insistent cependant aussi sur le fait que cette interjection est composée de deux impératifs (de deuxième personne) de deux verbes distincts: «di » du verbe «dire» et «va»du verbe «aller».

Les graphies que l'on rencontre dans les éditions de textes anciens reflètent également ce questionnement et témoignent d'un certain dilemme chez les éditeurs modernes: on y rencontre en effet «di va» en deux mots, comme dans [I] :

[I] Quant li rois ot mengié, si l’a a raison mis. / «Di va! dist Alixandres, dont es? de quel païs?» / Li harperres respont: «Sire, tu as mespris; / [...].»

Après avoir mangé, le roi lui a adressé la parole. «Dis-moi, dit Alexandre, d'où es-tu? De quel pays?» Le musicien lui répond: «Seigneur, tu as mal agi; [...].»

(Le Roman d'Alexandre, br. I, v. 2635-2637)

aussi bien que «diva» en un seul mot, comme dans [2]:

1. Cette contribution s'inscrit dans le projet de recherche FFI20I0-15158/FILO, financé par le Ministerio de Ciencia e Innovación, Espagne (Plan Nacional I+D+i 2008-20II).

2. En s'appuyant sur la terminologie de Bühler, Sirdar Iskandar (1983: 3I) distingue ainsi les «interjections d'appel» (comme allons!), destinées à agir sur l'interlocuteur, et les marqueurs subjectifs (comme bélas!), ayant une «fonction d'expression» et exprimant par conséquent un état psychologique du locuteur. 
[2] A l'ostel vint, en haut s'escrie: / «Diva! Sont cuites les pertris? / - Sire, dist ele, ainçois va pis! / Quar mengiés les a li chas.»

De retour à la maison, il [le paysan] s’écria à haute voix: «Alors? Les perdrix sont-elles cuites? - Seigneur, dit-elle, quel grand malheur! En effet, le chat les a mangées.»

(Le Dit des perdriz, v. 56-59)

Par ailleurs, le rapprochement proposé par Greimas et Buridant avec les interjections «allons» et «dis donc» ${ }^{3}$ pose bien sûr aussi la question du sens que l'on peut attribuer à «di va»: face à une langue ancienne, dont il n'existe par conséquent plus de locuteur natif, seuls des critères contextuels et distributionnels peuvent nous éclairer sur ce point, notamment la classe d'équivalence dans laquelle entre «di va».

On note à ce propos que «di va» semble se trouver dans le même type de contextes que «di» (employé seul) ou «di moi» et sa variante «or me di» (signifiant «dis-moi donc»). C'est ce que montrent les exemples [3a] à [3c], où l'impératif «di» est suivi, tout comme «di va» dans [I] et [2], d'une proposition interrogative:

[3a] «Di, feras-tu autre cose?»

«Dis, feras-tu autre chose?»

(Gautier d'Arras, Eracle, v. 5991)

[3b] «Renart, faist il, biax douz conpaig, / di moi, ies tu or ça dedanz?»

«Renart, dit-il, mon cher compère, dis-moi, es-tu à présent là à l'intérieur?»

(Le Roman de Renart, br. I, v. 784-785)

[3c] «Or me di, frere debonaire, / ces armes, qui les te bailla?»

«Dis-moi donc, noble frère, ces armes, qui te les a données?»

(Chrétien de Troyes, Le Conte du graal (Perceval), v. 1372-1373)

Mais parallèlement à ce type d'occurrences, «di va» apparaît aussi dans des contextes déclaratifs ou injonctifs, dans lesquels une commutation avec «di» ou «di moi» devient impossible. C'est ce qu'attestent [4] et [5]:

[4] El regarder qu'il fist l'ymage, / Amors ralume son corage, / se li dist: «Or aies envie: / ci en maine Paris s'amie. / (Ha! Dieus! Verrai jou ja le jor / k'ensi en maigne Blanceflor?) / Diva, Floires! Aprés mangier / te doit tes ostes consillier.»

Tandis qu'il contemplait cette scène, Amour ralluma la flamme dans son cour: «Prends exemple sur Pâris que tu vois ici emmener sa bien-aimée! - Ah! Dieu!

3. Vincensini (2006: IO2, note I) propose de traduire «diva» par «dis, allez», traduction qui met l'accent sur l'origine «double» de cette interjection. 
Pourrai-je voir le jour où j'emmènerai pareillement Blanchefleur? - Allons, Floire, après le repas, ton hôte doit t'aider de ses conseils.»

(Robert d'Orbigny, Le Conte de Floire et Blanchefleur, v. 1707-1714)

[5] Molt fu [Volcens] iriez, s'espee trait, / halça le cop por lui [le danzel] ferir; / donc ne se pot Nisus tenir, / mais a la mort s'abandona, / sailli avant, se li cria. / «Di va», fait il, «ne lo tochiez, / mais moi prenez, si vos vanchiez.»

Très en colère, il tira son épée, la leva pour mieux frapper le jeune homme; alors Nisus ne put se retenir, il s'abandonna à la mort, il sauta en avant et lui cria: «Allons, ne le touchez pas, mais prenez-moi et vengez-vous.»

(Eneas, v. 5220-5226)

On peut alors se demander si, dans les différents types de contextes dans lesquels nous rencontrons «di va», nous avons toujours affaire à la même interjection, au même marqueur discursif?

Notre étude tentera de répondre à ces différentes interrogations. Elle s'inscrit dans le cadre de la grammaticalisation au sens large, et plus précisément dans le cadre de la pragmaticalisation, telle qu'elle est notamment présentée dans les travaux de Dostie (2004: 27): les marqueurs discursifs issus de formes verbales y sont considérés comme le résultat d'un processus de pragmaticalisation verbale, par lequel ces formes acquièrent progressivement un rôle au plan discursif. Il s'agira donc, entre autres, de s'interroger sur les différents degrés de pragmaticalisation de «di» et de «va» que révèlent les emplois de «di va».

Pour ce faire, nous nous appuierons sur un corpus de 35 textes littéraires en vers ${ }^{4}$ datés des XII ${ }^{e}$ et XIII ${ }^{e}$ siècles et comportant l'interjection «di va» et/ou des occurrences de «di»/ «va» en emploi absolu. Ce corpus nous a permis de relever 58 occurrences de «di va» / " diva» interjectif, dont 30 se trouvent en contexte interrogatif, I6 en tête d'un énoncé impératif et 12 en contexte déclaratif ${ }^{5}$.

On s'intéressera ainsi dans un premier temps, aux cas de cooccurrence de «di» et de «va» et au figement de la tournure «di va», puis on abordera la question de la pragmaticalisation de l'impératif «di» dans cette tournure. Enfin seront examinés la position et le rôle de «di va» à l'intérieur du dialogue.

\section{Les cas de cooccurrence de «di » et de «va » et le figement de la tournure «di va»}

Pour appréhender la valeur de "di va», il est tout d'abord possible de s'interroger sur le lien que la juxtaposition de ces deux impératifs entretient avec la séquence dans

4. L'emploi de «di va» semble en effet lié à la forme versifiée: les romans en prose que nous avons consultés n'en comportent aucune occurrence.

5. Dans près de la moitié des occurrences, «di va» est par conséquent employé dans un contexte autre qu'interrogatif. 
laquelle la forme verbale de «aller» précède celle de «dire». Une telle comparaison nous aidera en effet à évaluer si l'ordre «di» + «va» témoigne d'un figement particulier par rapport à «va»+ «di».

\section{1. «Va » précède « di »}

Lorsque, dans un énoncé, l'impératif «va» se trouve directement suivi de l'impératif «di», l'on remarque que seul le verbe «aller» est employé de manière absolue. En effet, «di» y comporte toujours au moins un complément, ce qui rend la formation d'une unité syntaxique «* va di» impossible. Deux cas de figure se présentent alors.

D'une part, «va» et «di» peuvent traduire deux injonctions distinctes. Cela se produit en particulier lorsque le locuteur fait de son allocutaire un messager, dans la mesure où celui-ci doit dans un premier temps «aller» auprès du destinataire du message avant de «dire», de transmettre le message en question; c'est le cas dans [6] :

[6] «Va, di Wedon a la fiere vertu, / le mien chier frere qi le poil a chenu, / q'il viegne a moi [...].»

«Va dire à Eudes au cour valeureux, mon cher frère aux cheveux blancs, qu'il vienne à moi [...].»

(Raoul de Cambrai, v. 1787-1789)

Cet énoncé est repris, dans la laisse suivante, par [7] :

[7] «Va, si me di mon frere dant Wedon / q'il vaigne a moi [...].»

«Va dire à mon frère Eudes qu'il vienne à moi [...].»

(Raoul de Cambrai, v. 1794-1795)

où l'emploi de l'adverbe de liaison «si» (que l'on pourrait traduire ici par la conjonction «et») met bien en lumière le fait que les deux impératifs traduisent des injonctions distinctes.

Mais «va» + «di» est aussi en mesure d'exprimer un seul ordre, celui de dire quelque chose. Dans ce cas, le contexte de l'occurrence permet de déduire qu'aucun déplacement n'est nécessaire à l'allocutaire pour «dire» ce que souhaite le locuteur : la forme «va» a alors perdu son sens premier. Nous en avons des exemples dans [8] et [9] :

[8] «Je le [le cheval] vandrai, Tibert a dit. / - Et por conbien, Renart a dist, / le doras tu? va, di le moi.»

«Je vais le vendre, a dit Tibert. - Et pour combien, a dit Renart, me le donneras-tu? Allez, dis-le-moi.»

(Le Roman de Renart, br. XI, v. 12147-12149) 
[9] Tote voie tant m'anhardi, / que je li dis: «Va, car me di / se tu es boene chose ou non.»

Toutefois, je m'enhardis assez pour lui dire: «Hé, dis-moi donc si tu es ou non une bonne créature.»

(Chrétien de Troyes, Le Chevalier au lion (Yvain), v. 325-327)

Ces deux exemples impliquent la coprésence des interlocuteurs: l'allocutaire de «va»+ «di» n'est donc pas censé se déplacer; il doit seulement répondre à la question du locuteur. Par conséquent, «va» doit y être considéré comme un marqueur discursif ${ }^{6}$, statut qui peut d'ailleurs également lui être attribué dans des occurrences où «di» est absent:

[Io] Qui es tu, va, qui vas par ci?

Qui es-tu, hé! toi, qui vas par ici?

(Rutebeuf, Le Miracle de Théophile, v. 540)

Ce constat nous permet de faire l'hypothèse - à vérifier dans une étude ultérieure - que le développement du marqueur discursif «va» est antérieur à celui de «di» interjectif, dans l'histoire du français. Même en emploi absolu, «di» semble en effet, pendant la période de l'ancien français, toujours rester compatible avec le maintien de son sémantisme premier.

Notons enfin que dans les exemples que nous venons de citer ([6] ̀̀ [9], ainsi que [io]), l'emploi de «va» + «di» va toujours de pair avec le tutoiement de l'allocutaire.

\section{2. «Di » précède «va »}

Lorsque nos deux impératifs forment une séquence «di» + «va», seul un exemple se rapproche des occurrences que nous venons de citer; il s'agit de [II] :

[iI] LI AMIRAUS D'ORQUENIE: [...] A genous le trouvai ourant, / A jointes mains et en plourant, / Devant sen cornu mahommet. / LI ROIS : Di, va, vilains, se tu $i$ crois. / LI PREUDOM: Oil, sire, par sainte crois; / [...].

Je l'ai trouvé agenouillé, en prières, mains jointes et en larmes, devant son fétiche cornu. - Allons! misérable, dis-moi si tu y crois. - Oui, seigneur, par la sainte croix!

(Jehan Bodel, Jeu de saint Nicolas, v. 511-515)

Ici, «di» est complété, comme dans [9], par une subordonnée percontative ${ }^{7}$ «se $\mathrm{P}$ »: il s'agit donc d'un véritable impératif injonctif, alors que «va» n'implique pas de déplacement de la part de l'interlocuteur et devient marqueur discursif.

6. [8] et [9] soulignent alors une différence entre le «va» interjectif de l'ancienne langue et celui du français moderne. Comme le montre Sierra Soriano (2006: 74-75), l'interjection «va» figure de nos jours en postposition (Courage, va!) ou se trouve associée à un autre élément (Allez, va! - Va donc!).

7. Nous empruntons ce terme à P. Le Goffic (I994: 42-44). 
Mais, du point de vue syntaxique, l'énoncé [II] représente une exception à l'intérieur de notre corpus. Dans toutes les autres occurrences de la séquence «di» + «va», les deux verbes sont en effet employés de manière absolue. Ils ne traduisent alors jamais deux injonctions distinctes, le sens de base du verbe «aller» n'y étant jamais conservé. Comme dans [8], [9] et [II], ils peuvent correspondre à la demande de «dire» quelque chose. C'est notamment le cas dans [I] et [2] :

[I] Quant li rois ot mengié, si l’a a raison mis. / «Di va! dist Alixandres, dont es? de quel païs?»

(Le Roman d'Alexandre, br. I, v. 2635-2636)

[2] A l'ostel vint, en haut s'escrie: / «Diva! Sont cuites les pertris?»

(Le Dit des perdriz, v. 56-57)

Mais «di va» est par ailleurs également compatible avec des contextes dans lesquels la valeur de base de «dire» s'estompe à son tour; nous l'avons déjà constaté avec les exemples [4] et [5]:

[4] «Diva, Floires! Aprés mangier / te doit tes ostes consillier.»

(Robert d'Orbigny, Le Conte de Floire et Blanchefleur, v. 1713-1714)

[5] «Di va», fait $\mathrm{il}$, «ne lo tochiez, / mais moi prenez, si vos vanchiez.»

(Eneas, v. 5225-5226)

On le voit aussi dans [ı2], où «di va» est suivi de l'impératif du verbe «soi taisir» (se taire).

[i2] LI PREUDOM: Sains Nicolais, le tien secours! / [...] / Sains Nicolais, car me regarde! / Je me sui mis en vostre garde, / [...] / LI ANGELES: Diva! biaus crestiens, tais te, ne pleure! / De che dont iés desous seras deseure.

Saint Nicolas, à l'aide! [...] Saint Nicolas, regarde-moi donc! Je me suis mis sous votre protection [...] - Allons, cher chrétien, tais-toi, ne pleure pas! Ce qui t'accable, tu en triompheras.

(Jehan Bodel, Jeu de saint Nicolas, v. 1256-1263)

L'exemple [5] nous montre par ailleurs que, contrairement à ce que nous avions noté au sujet de «va»+ «di», «di va» est compatible avec un discours à la cinquième personne et n'implique donc pas forcément le tutoiement de l'interlocuteur, ce qui témoigne d'une certaine décatégorisation des deux formes.

Ces différents traits permettent de percevoir «di»+ «va» comme étant plus figé que «va»+ «di»: c'est uniquement avec «di»+ «va» que les impératifs sont tous les deux employés de manière absolue, peuvent tous les deux perdre leur valeur sémantique première et témoigner d'une certaine décatégorisation. Des occurrences 
comme [4], [5] et [I2], où l'on fait précisément ce constat, nous invitent alors à considérer «di va» non pas comme la juxtaposition de deux impératifs mais comme «une interjection», qui sert prioritairement à interpeller l'allocutaire.

\section{Les degrés de pragmaticalisation de l'impératif « di »}

Un problème particulier reste, en revanche, posé par les occurrences de «di va» où seul le sémantisme premier de «va» est perdu. Comment les différencier par exemple de [8] et [9], où «va» a cette même propriété?

Se pose alors plus précisément la question de la pragmaticalisation de l'impératif «di». Est-ce que le non-effacement du sémantisme premier de «dire», que nous avons observé en contexte interrogatif, nous amène forcément à y analyser le «di» de «di va» comme un véritable impératif injonctif comme dans [8] et [9]?

\subsection{Une première hypothèse et ses limites}

Il est vrai que, face à nos premières observations, on est tenté de distinguer deux cas de figure.

D'un côté, on regrouperait les «di va» figurant en contexte interrogatif, où il est donc demandé à l'allocutaire de dire quelque chose, de l'autre, les «di va» employés en contexte déclaratif ou injonctif, où il n'y a plus de demande de dire et où le sémantisme premier de «di» s'efface par conséquent.

Dans le premier cas, nous avons déjà noté les commutations possibles de «di va» avec «di», «di moi» et «or me di», commutations qui deviennent impossibles dans le deuxième cas.

On pourrait donc en déduire qu'en contexte déclaratif et injonctif, «di va» représente bien «une interjection» constituée elle-même de deux marqueurs discursifs, «di» et «va». En contexte interrogatif en revanche, seul «va», ayant perdu son sens premier, serait considéré comme pragmaticalisé et pourrait alors être assimilé à un marqueur discursif qui porte sur l'impératif injonctif «di».

Mais cette analyse a des limites. En effet, si l'on examine les occurrences dans lesquelles «di va» figure en contexte interrogatif, on y relève aussi plusieurs indices qui mettent en question l'analyse de «di» comme véritable impératif injonctif.

Tout d'abord, «di va» y est compatible avec le vouvoiement de l'interlocuteur, ce qui témoigne donc du fait que «di» n'y est pas forcément perçu comme une forme verbale de deuxième personne. C'est ce qui se produit dans [I3] et [I4]:

[13] Il les araisona premier. / «Di va» fait il, «qui estes vos, / qui an cest regne tenebros / armé vos estes anbatu? / Dites, por coi estes venu? / [...].»

Il leur parla en premier. "Dites donc, dit-il, qui êtes-vous, vous qui vous êtes précipités en armes dans ce royaume ténébreux? Dites, pourquoi êtes-vous venus?» (Eneas, v. 2510-2513) 
[I4] «[...] / Qu'est-ce, sire prestres? Diva, / me volez vous plus traveillier?»

«Qu'est-ce que c'est, messire le prêtre? Dites donc, vous voulez m’épuiser encore plus?»

(D'Estourmi, v. 524-525)

Par ailleurs, «di va» peut être suivi de l'impératif «di» régissant une proposition percontative. On peut en déduire que le «di» de «di va» n'exprime pas, dans ce cas, l'injonction de «dire» et n'est par conséquent pas perçu comme un véritable impératif (ce qui autorise à faire l'hypothèse d'une perte de son sémantisme premier dans ces contextes):

[r5] Dit Tibert: «Tu es trop musart. / - Diva or di, ce dit Renart, / N'en estas-tu legier assez? / - Ce dit Tibers: Vos i gabez.»

Tibert dit: «Tu es vraiment sot. - Allons, reprit Renart, dis-moi donc, n'en [du fait de ne plus avoir de queue] es-tu pas considérablement allégé? - Tibert répondit: Vous vous moquez (de moi).»

(Le Roman de Renart, br. XIV, v. 125-128)

Et si nous avons vu que «di va» commute, en contexte interrogatif, avec «di» et ses variantes, on peut noter que cette séquence commute aussi avec le marqueur discursif «va», qui ne comporte en aucun cas le sème de [dire]. Comparons [I] avec [I6]:

[I] Quant li rois ot mengié, si l’a a raison mis. / «Di va! dist Alixandres, dont es? de quel païs?»

(Le Roman d'Alexandre, br. I, v. 2635-2636)

[16] Il vint au roi, et il l'apele: / «Va, dont viens tu? - J'aport novele / [...].»

Il s'approche du roi et celui-ci l'interpelle: «Hé! D’où viens-tu? - J'apporte des nouvelles [...].»

(Béroul, Le Roman de Tristan, v. 3389-3390)

et $[\mathrm{I} 7]$ avec $[\mathrm{Io}]$ :

[17] Dont si li dist: «Que fais tu la, / diva! Caitis mal eürés? / [...].»

Alors il lui dit: «Que fais-tu là, dis, pauvre malheureux?»

(Gautier d'Arras, Eracle, v. 5944-5945)

[Io] Qui es tu, va, qui vas par ci?

(Rutebeuf, Le Miracle de Théophile, v. 540)

\subsection{Distinction entre «di va 1 » et « di va 2 »}

Ces différents constats nous amènent à distinguer finalement trois cas de figure, dans lesquels nous rencontrons la séquence «di» + «va». 
Dans un premier type d'occurrences, l'impératif «di» est accompagné du marqueur discursif «va»: nous limitons cette analyse aux occurrences dans lesquelles le COD de «di» est exprimé. L'ordre des deux éléments n'est alors pas fixe : on rencontre «va»+ «di» [8] et «di»+ «va» [II], bien que ce deuxième cas semble plus rare que le premier. Et «di» implique toujours le tutoiement de l'allocutaire. Nous laisserons de côté ce type d'occurrences injonctives dans la suite de cette étude.

Dès que «di» et «va» sont tous deux employés de manière absolue, on a vu que leur ordre se fixe et qu'il y a compatibilité avec un discours à la cinquième personne. Nous considérons de ce fait «di va» dans ce cas comme un marqueur discursif complexe, en admettant une certaine polysémie de celui-ci.

On appellera «di va 2» l'emploi le plus évident, où le contexte de «di va» comporte des indices permettant de poser la perte du sémantisme premier des deux formes verbales : cela concerne en premier lieu les contextes déclaratifs et injonctifs, mais aussi certains contextes interrogatifs comme [13] et [I4]. Et on appellera «di va I» les marqueurs qui restent compatibles avec le maintien du sens premier du verbe «dire», comme c'est le cas dans [I] et [2].

Cette distinction entre «di va 2 » et «di va I» peut être rapprochée de la différenciation établie par Waltereit (2007) entre marqueurs discursifs complexes composés exclusivement de marqueurs discursifs clairement définis (c'est le cas de «di va 2 ») et marqueurs discursifs complexes composés d'un marqueur discursif et d'un autre élément. On pourrait analyser ainsi «di va I», marqueur pour lequel il est en effet difficile de déterminer le statut exact de «di»: cette forme étaitelle encore réellement perçue par les locuteurs de l'époque comme un impératif traduisant une demande de dire quelque chose, ou était-elle déjà assimilée à un marqueur attirant l'attention de l'allocutaire sur l'interrogation qui suit? La même question se pose d'ailleurs aussi pour les occurrences dans lesquelles «di» figure seul - cf. [3a] à $[3 \mathrm{c}]^{8}$. Sur ce point, il nous semble possible de faire l'hypothèse d'une superposition des deux interprétations en cause, en partant du principe que, comme le souligne d'ailleurs Dostie, la pragmaticalisation d'une unité n'implique pas forcément, du moins dans ses étapes intermédiaires, la perte du sémantisme premier de l'unité concernée ${ }^{9}$. Dans cette perspective, le «di» de «di va I» témoignerait, tout comme celui des occurrences citées sous [3], déjà d'un début de pragmaticalisation.

8. Pitavy (2005: 150) parle dans ce cas d'«auxiliaire injonctif d'interrogation», terminologie qui souligne bien le double statut de «di» dans ce type de contexte: tout en gardant sa valeur injonctive, l'impératif y devient «auxiliaire» de l'interrogation qu'il accompagne.

9. Il s'agit du principe de persistance: «dans les stades intermédiaires où il y a pragmaticalisation, on peut s'attendre à ce qu'une forme soit polysémique et à ce qu'un ou plusieurs sens reflètent le sens d'origine» (Dostie, 2004: 35). 


\section{La position du marqueur discursif « di va » à l'intérieur du discours}

Si l'on s'interroge à présent sur la position et sur le rôle de «di va» à l'intérieur du discours, les occurrences que nous venons de voir montrent que «di va I», tout comme «di va 2 », se rencontrent principalement en position initiale, donc dans une configuration «Di va, $\mathrm{P} »$.

\section{1. «Di va » comme introducteur d'un tour de parole}

Pour être plus précis, on peut dire que «di va» se trouve en principe à l'initiale d'un tour de parole. Il y a alors deux possibilités. Soit «di va, $\mathrm{P}$ » ouvre le dialogue, comme par exemple dans [2] et [13]:

[2] A l'ostel vint, en haut s'escrie: / «Diva! Sont cuites les pertris?»

(Le Dit des perdriz, v. 56-57)

[13] Il les araisona premier. / «Di va» fait il, «qui estes vos, / qui an cest regne tenebros / armé vos estes anbatu? / Dites, por coi estes venu? / [...].»

(Eneas, v. 2510-2513)

Soit cette séquence se trouve à l'intérieur du dialogue et indique alors un changement de tour de parole, comme on le constate dans [I2] et [I5].

[12] LI PREUDOM: Sains Nicolais, le tien secours! / [...] / Sains Nicolais, car me regarde! / Je me sui mis en vostre garde, / [...] / LI ANGELES: Diva! biaus crestiens, tais te, ne pleure! / De che dont iés desous seras deseure.

(Jehan Bodel, Jeu de saint Nicolas, v. 1256-1263)

[I5] Dit Tibert: «Tu es trop musart. / - Diva or di, ce dit Renart, / N'en estas-tu legier assez?» (Le Roman de Renart, br. XIV, v. 125-127)

Dans tous ces cas, «di va» participe, avec d'autres éléments tels que les termes d'adresse ${ }^{10}$, d'autres interjections ${ }^{11}$ et les verbes de parole employés dans le discours citant $^{12}$, à la signalisation du discours rapporté direct. En l'absence de guillemets et de tirets dans les manuscrits médiévaux, on peut imaginer que «di va» constitue un des «signaux» pour le lecteur lui indiquant le passage du récit au discours direct

10. Leur position à l'intérieur du discours direct, et notamment en relation avec l'emploi des verbes de parole, a été étudiée par Lagorgette (2004).

11. Le rôle structurant des interjections dans les discours rapportés de la prose médiévale a été souligné par Vincensini (2006: IO3-IO4).

12. Concernant la position et le rôle des verbes de parole dans la signalisation du discours direct, en relation avec le genre littéraire dont il relève, cf. Marnette (2006: 37-40). 
ou le passage d'une réplique à une autre. On peut comprendre de cette manière l'exemple [I8], si on accepte de changer la ponctuation (introduite par l'éditeur moderne), en plaçant le second point d'interrogation à la fin du premier vers:

[18] Diva, ce qu'est? comant a non, / Diva? Andoille l'apelle on.

(Le Roman de Renart, Supp., v. 393, cité d'après le dictionnaire de Godefroy)

Ce type d'indice est aussi une aide précieuse pour comprendre le dialogue de [4], qui n'est pas signalé comme tel dans l'édition reproduite ici:

[4] El regarder qu'il fist lymage, / Amors ralume son corage, / se li dist: «Or aies envie / / ci en maine Paris s'amie. / (Ha! Dieus! Verrai jou ja le jor / k'ensi en maigne Blanceflor?) / Diva, Floires! Aprés mangier / te doit tes ostes consillier.»

(Robert d'Orbigny, Le Conte de Floire et Blanchefleur, v. 1707-1714)

Si le début du discours direct y est bien attribué au dieu Amour, l'énoncé entre parenthèses introduit par «Ha! Dieus!» exprime en revanche les inquiétudes de Floire, inquiétudes qu'Amour cherche à dissiper en reprenant la parole: «Diva, Floires! [...]».

Notons enfin que le changement de tour de parole signalé par «di va» n'implique pas forcément un changement de locuteur. On le voit dans [19]:

[19] Eracles le [la pierre] coisist de loing / et de si loing que il le voit / traist soi vers celui qui l'avoit, / [...] / «Or ai, fait il, ma desiree! [...] / [...] / or ne crien je fors Diu nului. / Diva, fait il dont a celui, / Est ce te piere? Vent le moi. / - Sire, ele est moie et je par foi le vous vendrai molt volentiers, / [...].»

Eracle l'aperçut de loin, et, d'aussi loin qu'il la vit, il se dirigea vers celui qui l'avait [...] «Maintenant, dit-il, j'ai ce que je désire! [...] À présent je ne crains personne hormis Dieu. Dis donc, dit-il alors à l'homme, est-ce ta pierre? Vends-la-moi. - Seigneur, elle est à moi, et, par ma foi, je vous la vendrai très volontiers.»

(Gautier d'Arras, Eracle, v. 846-863)

où «di va» marque le passage d'un monologue commençant par «Or ai, fait il, ma desiree!», au discours qu'il adresse au vendeur de la pierre précieuse: «Diva, fait il dont a celui, / Est ce te piere?».

\subsection{La position de «di va» en contexte interrogatif}

À côté de ces observations, on note toutefois aussi qu'en contexte interrogatif, «di va» ne se trouve pas exclusivement à l'initiale d'un tour de parole: «di va $+\mathrm{P}$ » peut alors en effet se trouver à l'intérieur d'un tour de parole, comme dans [I4], ou encore, «di va» peut suivre $\mathrm{P}$, comme dans [I7].

[I4] «[...] / Qu'est-ce, sire prestres? Diva, / me volez vous plus traveillier?»

(D’Estourmi, v. 524-525) 
[17] Dont si li dist: «Que fais tu la, / diva! Caitis mal eürés? / [...].»

(Gautier d'Arras, Eracle, v. 5944-5945)

13. Selon Roulet (I98I), l'intervention «initiative» et l'intervention «réactive» constituent la structure de l'échange (qui est considéré comme l'unité de base du dialogue): la première intervention «initie» l'échange et appelle une réponse, qui est apportée par l'intervention dite «réactive». Ce binôme peut être illustré par les couples «question/réponse» et «injonction/refus ou acceptation». 
types, en particulier lorsque l'interrogative introduite par «di va» constitue une demande de confirmation. Prenons comme exemple [20], où l'affirmation «Athis est pris» entraîne la demande «Di va, $\mathrm{P}$ ?»:

[2o] «Sire, fait il, Athis est pris. / [...]» / Procelias l'a regardé; / Son cheval a tost arresté, / Mue coulor et devint noirs: / «Di va, fait il, puet estre voirs / Qu'il aient pris mon compaignon? / Sés tu se il est voirs ou non?» / Cil li respont qu'il le vit prendre / [...].

«Seigneur, dit-il, Athis est pris [...]» Procelias le regarda et freina aussitôt son cheval, il changea de couleur et devint blême: «Allons, dit-il, cela peut-il être vrai qu'ils aient pris mon compagnon? Sais-tu si c'est vrai ou non?» L'autre lui répondit qu'il en fut témoin [...].

(Le Roman d'Athis et de Procelias, v. 4294-4302)

De manière générale, les emplois réactifs représentent, dans notre corpus, 22,5\% des «di va I» et $67 \%$ des «di va 2 ». Dans ces emplois à valeur réactive, «di va, $\mathrm{P}$ » réagit souvent à l'énoncé attribué à un premier locuteur, comme on vient de le voir pour [20] et pour [I2]. C'est aussi le cas dans [2I], où Guillaume réagit aux consignes de Bertrand:

[2I] Et dit Bertran: «A maleiçon Dé! / Vo droit seignor ne devez pas haster, / Ainz le devez servir et hennorer, / Contre toz homes garantir et tenser. - Di va, fet il, ja m'a il si mené / Qu'a lui servir ai mon tens si usé; / N'en ai eü vaillant un oef pelé.»

Bertrand s'écrie: «Malédiction de Dieu! Vous ne devez pas provoquer votre légitime seigneur, mais le servir et l'honorer, le protéger et le défendre contre tous les hommes. - Allons donc! répond Guillaume, il m’a amené à un point tel qu’à le servir j’ai passé tout mon temps, et je n'en ai pas reçu la valeur d'un œuf sans coquille.»

(Le Charroi de Nîmes, v. 421-427)

Mais «di va» peut aussi réagir en premier lieu à l'acte d'énonciation que vient d'accomplir le premier locuteur. On en a un exemple dans [22], où le chevalier signifie à l'écuyer qu'il n'avait pas à lui poser de question sur son manque de combativité:

[22] [...] et [l'écuyer] dist: «Vasax, / dont n'iestes vos sains et heitiez, / qui ci tote jor agaitiez / et nule rien n'i avez fete, / escu troé ne lance frete? / - Di va, fet $i l$, a toi que taint? / [...] / [...] ce n'iert mie ore, / que dire nel te daigneroie.»

[...] et il dit: «Chevalier, seriez-vous en mauvaise santé pour rester ainsi toute la journée aux aguets sans rien faire, ni transpercer un écu ni rompre une lance? - Allons, réplique l'autre, en quoi cela te regarde? [...] ce n'est pas aujourd'hui que je daignerais te le dire.»

(Chrétien de Troyes, Le Conte du graal (Perceval), v. 5110-5119)

Dans un dernier type d'occurrences, «di va, $\mathrm{P}$ » exprime une réaction face au comportement non verbal de l'interlocuteur, comme c'est le cas dans [5]: 
[5] Molt fu [Volcens] iriez, s'espee trait, / halça le cop por lui [le danzel] ferir; / donc ne se pot Nisus tenir, / mais a la mort s'abandona, / sailli avant, se li cria. / «Di va», fait il, «ne lo tochiez, / mais moi prenez, si vos vanchiez.»

(Eneas, v. 5220-5226)

\subsection{Position et valeur expressive de « di va »}

Dans la mesure où «di va» peut introduire une intervention réactive, il ne convient pas de le considérer exclusivement comme une «interjection d'appel». Lorsqu'il réagit à une intervention, ou à un comportement, le marqueur discursif prend en effet inévitablement aussi une valeur expressive. Selon le contexte dans lequel il figure, il traduit alors différentes attitudes de son locuteur.

Il exprime ainsi une certaine bienveillance de la part du locuteur à l'égard de son interlocuteur dans $[4]$ :

[4] El regarder qu'il fist lymage, / Amors ralume son corage, / se li dist: «Or aies envie: / ci en maine Paris s'amie. / (Ha! Dieus! Verrai jou ja le jor / k'ensi en maigne Blanceflor?) / Diva, Floires! Aprés mangier / te doit tes ostes consillier.»

(Robert d'Orbigny, Le Conte de Floire et Blanchefleur, v. 1707-1714)

ainsi que dans [12] (cité en 4.3). Mais, dans la plupart des occurrences, il souligne un refus, un désaccord, une opposition du locuteur par rapport à ce que vient de dire ou de faire son interlocuteur: nous l'avons vu dans [5] ainsi que dans [2I] et [22]. Enfin, «di va» peut traduire la surprise, voire l'incrédulité du locuteur, notamment lorsqu'il introduit une demande de confirmation comme dans [20].

On comprend alors mieux pourquoi la tournure «Di va, $\mathrm{P}$ » en initiale de tour de parole, qui domine de manière générale, s'impose néanmoins davantage pour certains emplois de «di va» que pour d'autres, autrement dit pourquoi on la rencontre plus systématiquement avec «di va 2 » qu'avec «di va I».

La position initiale est certes fréquente pour les «di va» correspondant à des «interjections d'appel», faisant appel à l'attention de l'allocutaire. Mais, comme nous l'avons constaté, on peut rencontrer ces «di va» aussi dans d'autres positions, notamment à la suite de $\mathrm{P}$. Dans tous ces cas, «di va» attire alors l'attention de l'allocutaire sur son contexte discursif immédiat. Or, ces «interjections d'appel» sont essentiellement des «di va I».

La position initiale s'avère davantage incontournable pour les «di va» qui prennent également une valeur expressive, ce qui est le cas dans la majorité des «di va 2 »: étant donné qu'il traduit une réaction du locuteur par rapport au contexte précédent, «di va» doit alors directement être rattaché à celui-ci. On peut dans ce cas même aboutir à des occurrences comme [23] :

[23] «[...] / Deus mantieus affublés se chaufoit a un feu. / - Di, va! Ce dist Porrus, et ja est il estés / Et fait si grant chalor com vos veoir pöés ; / [...].» 
«[...] couvert de deux manteaux, il [le roi] se chauffait au feu - Comment? répond Porus, mais c'est pourtant l'été, et vous pouvez bien voir quelle chaleur il fait.»

(Le Roman d'Alexandre, br. III, v. 1574-1576)

où «di va» est essentiellement, voire exclusivement, tourné vers son contexte gauche, en exprimant la surprise du locuteur face à l'affirmation qui précède.

\section{Conclusion}

Les éléments d'analyse qui précèdent nous ont permis de proposer une description plus précise d'une interjection propre à l'ancien français qui reste peu étudiée par les médiévistes, tout en l'abordant sous un angle nouveau, en tant que marqueur discursif. Nous avons ainsi établi la distinction entre deux catégories d'emplois de «di va» («di va I» et «di va $2 »)$, distinction qui résulte en priorité du degré de pragmaticalisation plus ou moins élevé de la forme «di», mais qui correspond également à des distributions différentes du marqueur à l'intérieur du tour de parole et de l'échange. Les propriétés contextuelles distinctes de «di va I» et de «di va 2» dans le dialogue, mettent alors en évidence que, contrairement à ce que peut laisser supposer la nature d'origine des éléments qui le composent (il s'agit d'impératifs), notre marqueur discursif (et plus précisément «di va $2 »)$ ne représente pas exclusivement une interjection d'appel centrée sur l'allocutaire. Sa position en tête d'intervention réactive en fait aussi un marqueur à valeur expressive, qui rend compte de la subjectivité de son locuteur.

\section{Bibliographie}

BURIDANT, C. 2000. Grammaire nouvelle de l'ancien français. Paris: SEDES.

Buridant, C. (ed.) 2006. Langages: L'interjection: jeux et enjeux i6r. Paris: Armand Colin.

Dostie, G. 2004. Pragmaticalisation et marqueurs discursifs. Analyse sémantique et traitement lexicographique. Champs linguistiques. Bruxelles: De Boeck-Duculot.

Dostie, G. et Pusch, C.D. (eds) 2007. Langue française: Les marqueurs discursifs I54. Paris: Armand Colin.

Greimas, A.J. 1980. Dictionnaire de l'ancien français jusqu'au milieu du XIVe siècle. Paris: Larousse.

LAGorgetTE, D. 2004. Termes d'adresses et verbes de parole en moyen français: une approche pragmatique. In J.M. Lopez Munos et al. (eds), Le discours rapporté dans tous ses états. Paris: L'Harmattan: 194-203.

Lagorgette, D., Oppermann-Marsaux, E. et Rodríguez Somolinos, A. (eds) 2006. Langue française: Énonciation et pragmatique: approche diachronique 149. Paris: Armand Colin.

Le Goffic, P. 1994. Grammaire de la phrase française. Paris: Hachette. 
MARnette, S. 2006. La signalisation du discours rapporté en français médiéval. Langue française $\mathrm{I} 49$ : $3 \mathrm{I}^{-} 47$.

OpPermann-Marsaux, E. 2008. De l'injonction à l'interjection: la naissance des marqueurs discursifs tien(s) et tenez en français médiéval (XII ${ }^{\mathrm{e}}-\mathrm{XV}^{\mathrm{e}}$ siècles). L'Information grammaticale II8: II-I5.

OPPERMANN-MARSAUX, E. 20IO. Injonction et interjection : l'évolution des emplois de l'impératif allons du moyen français jusqu'au français classique. In B. ComBETtes et al. (eds), Le changement en français. Études de linguistique diachronique. Berne - Berlin - Bruxelles: Peter Lang: 3II-324.

OpPERMANN-MARSAux, E. et Rodríguez Somolinos, A. (eds) 2008. L'Information grammaticale: Propriétés énonciatives et pragmatiques du texte médiéval II8. Louvain: Peeters.

Pitavy, J.C. 2005. Allez, dis donc... : dire entre verbe et opérateur du discours en grec, latin et français. Revue de Sémantique et Pragmatique 17: 137-150.

RoulEt, E. I98r. Échanges, interventions et actes de langage dans la structure de la conversation. Études de linguistique appliquée 44: 7-39.

SierRa Soriano, A. 2006. Interjections issues d'un verbe de mouvement: étude comparée français-espagnol. Langages I6r: 73-90.

Sirdar Iskandar, C. 1983. Allons! Semantikos 7 (I) : 28-44.

VINCENSINI, J.J. 2006. Formes et fonctions structurantes. À propos de quelques interjections en ancien et en moyen français. Langages I6I : IOI-II.

WALTEREIT, R. 2007. À propos de la genèse diachronique des combinaisons de marqueurs. L'exemple de bon ben et enfin bref. Langue française 154: 94-Io9.

\section{Textes cités}

Béroul. Le Roman de Tristan. Muret, E. (ed.) i979. CFMA i2. Paris: Champion.

Chrétien de Troyes. Le Chevalier au lion (Yvain). Roques, M. (ed.) i963. CFMA 89. Paris: Champion.

Chrétien de Troyes. Le Chevalier au lion (Yvain). Buridant, C. et Trotin, J. (trad.) i982. CFMA Traductions 5. Paris: Champion.

Chrétien de Troyes. Le Conte du graal (Perceval). Lecoy, F. (ed.) 1972. CFMA ioo. Paris: Champion. T. I. [T. 2: 1975. CFMA 103.]

D'Estourmi, in Fabliaux du Moyen Âge. Dufournet, J. (ed.) 1998. GF 972. Paris : Flammarion.

Eneas. Salverda de Grave, J.J. (ed.) 1983. CFMA 44. Paris: Champion. T. I. [T. 2: 1985. CFMA 62.]

Gautier d’Arras. Eracle. Raynaud de Lage, G. (ed.) i976. CFMA io2. Paris: Champion.

Jehan Bodel. Jeu de saint Nicolas. Henry, A. (ed.) 198I. Bruxelles: Académie royale de Belgique.

Le Charroi de Nîmes. Perrier, J.-L. (ed.) 1966. CFMA 66. Paris: Champion.

Le Charroi de Nîmes. GÉGou, F. (trad.) 1974. CFMA Traductions I4. Paris: Champion. 
Le Dit des perdriz, in Fabliaux du Moyen Âge. Dufournet, J. (ed.) 1998. GF 972. Paris: Flammarion.

Le Roman d'Alexandre. Armstrong, E.C. et al. (eds) et Harf-Lancner, L. (trad.) I994. Lettres gothiques. Paris: Librairie générale française.

Le Roman d'Atbis et de Procelias. Castellani, M.-M. (ed.) 2006. CFMA i5o. Paris: Champion.

Le Roman de Renart, br. I. RoQues, M. (ed.) i970. CFMA 78. Paris: Champion.

Le Roman de Renart, br. XI. Roques, M. (ed.) 1958. CFMA 85. Paris: Champion.

Le Roman de Renart, br. XIV, in Le Roman de Renart 2. Dufournet, J. et Méline, A. (eds) I99I. GF 4I9. Paris: Flammarion.

Raoul de Cambrai. Kay, S. (ed.) et KibleR, W. (trad.) 1996. Lettres gothiques. Paris: Librairie générale française.

Robert d’Orbigny. Le Conte de Floire et Blanchefleur. Leclanche, J.-L. (ed.) 2003. Champion classiques 2. Paris: Champion.

Rutebeuf. Le Miracle de Théophile. Dufournet, J. (ed.) i897. GF 467. Paris: Flammarion. 